\title{
Cereal-based dietary iron intervention for improvement of iron status in female runners
}

\author{
I. Alaunyte $^{1}$, V. Stojceska ${ }^{2}$, A. Plunkett ${ }^{3}$ and E. Derbyshire ${ }^{4}$ \\ ${ }^{1}$ School of Health Sciences, Liverpool Hope University, Liverpool L16 9JD, UK, ${ }^{2}$ Centre for Sustainable Energy Use in \\ Food Chains, Brunel University, London UB8 3PH, UK, ${ }^{3}$ Manchester Food Research Centre, Manchester Metropolitan \\ University, Manchester M15 6BH, UK and ${ }^{4}$ School of Healthcare Science, Manchester Metropolitan University, \\ Manchester MI 5GD, UK
}

Iron is an important micronutrient in energy production pathways and is essential for sufficient erythropoiesis. Female runners are at a higher risk of iron deficiency due to negative iron balance caused by insufficient iron intake and increased iron losses associated with endurance running ${ }^{(1)}$. Conventional treatments for iron deficiency include pharmacological supplements which often cause side effects including abdominal discomfort, constipation and nausea ${ }^{(2)}$. Iron fortified products have been successfully used to increase haemoglobin, serum ferritin levels and to reduce the risk of iron deficiency ${ }^{(3)}$. However, studies looking at developing and incorporating products naturally rich in iron are scarce. The aim of the study was to evaluate the effects of incorporating developed Teff cereal bread $^{(4)}$ into daily diets on dietary iron intake and iron status of recreational female runners.

Participants (n 11) completed a 6-week dietary intervention study. They were asked to consume 4 slices of iron-rich Teff bread as part of their daily diet. During this period, their dietary habits were assessed by multiple 24-hr recalls; iron status was determined by venous blood analysis for serum transferrin (sTRF), serum transferrin receptor (sTsfR), and serum ferritin (sFer).

Female runners reported inadequate daily dietary iron intake of $10.7 \mathrm{mg} /$ day at baseline, which was associated with reduced iron stores (sFer $r=0.7, P<0.05$ ). Over a third of all participants showed depleted bodily iron stores (sFer $<12 \mu \mathrm{g} / \mathrm{L})$. Dietary intervention resulted in significantly higher total iron intakes $(18.5 \mathrm{mg} /$ day, $P<0.05)$. Teff bread contributed to $38 \%$ of the daily iron intake. Non-significant improvements in iron tissue supply (sTRF 5.4\% change, sTsfR $12 \cdot 8 \%$ change, NS) and iron stores (sFer 5.3\% change, NS) were observed. The improvements in iron tissue supply indices were strongly associated with prolonged dietary intervention $(\Delta$ STRF $r=-0.7, P<0.05 ; \Delta$ sTsfR $r=-0.6, P<0.05)$ and compromised initial iron status $(\Delta \mathrm{sTRF} r=-0.7, P<0.05 ; \Delta \mathrm{sTsfR}$ $r=-0 \cdot 6, P<0 \cdot 05)$, whilst enlarged iron stored were associated with increased dietary iron intake $(\Delta \mathrm{sFer} r=0 \cdot 8, P<0 \cdot 05)$.

\begin{tabular}{lccl}
\hline \multirow{2}{*}{$\begin{array}{l}\text { Nutrients in Teff } \\
\text { bread }\end{array}$} & \multicolumn{2}{c}{ Amount per $100 \mathrm{~g}$} & $\begin{array}{l}\text { Contribution } \\
\text { to DRVs }\end{array}$ \\
\cline { 2 - 3 } & Mean & $\mathrm{SD}$ & $18 \%$ TEI \\
\hline Carbohydrate $(\mathrm{g})$ & $43 \cdot 2$ & $2 \cdot 32$ & $48 \%$ \\
Protein $(\mathrm{g})^{*}$ & $11 \cdot 0$ & $0 \cdot 02$ & $3 \%$ TEI \\
Fat $(\mathrm{g}) *$ & $3 \cdot 7$ & $0 \cdot 16$ & $50 \%$ \\
Fibre $(\mathrm{g})^{*}$ & $4 \cdot 5$ & $0 \cdot 32$ & $76 \%$ \\
Iron $(\mathrm{mg})^{*}$ & $5 \cdot 62$ & $0 \cdot 22$ & \\
\hline
\end{tabular}

Key: *experimentally determined

${ }^{1}$ Based on consumption of average daily portion of $200 \mathrm{~g}$ for female adult

aged $19-50$ years of age consuming 1,940 kcal/day

DRVs - Dietary Reference Values

TEI - total energy intake

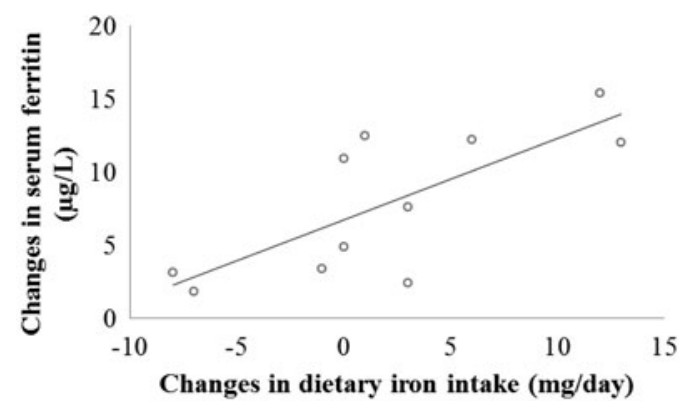

Fig. 1. Relationship between changes in dietary iron and serum ferritin $(r=0.8, P<0.05$ when controlling for dietary confounders, Partial Pearson's correlation

In conclusion dietary iron interventions using staple food products offer a promising way of improving dietary iron intake and overall iron status of female runners. Teff bread could be used as an iron-rich staple food alternative. It offers the opportunity to improve habitual dietary iron intakes. Favourable trends were observed between improved iron intakes and iron status in this study.

1. Burke LM, Millet GE and Tarnopolsky MA (2007) $J$ Sports Sci 25, S29-S38.

2. Hallberg L, Ryttinger L, Solverll L (1966) Acta Med Scand 459, 3-10.

3. Gera T, Sashdev HS and Boy E (2012) Am J Clin Nutr 96, 309-324.

4. Alaunyte I.Stojceska V, Plunkett A et al. (2012) J Cereal Sci 55, 22-30. 